\title{
Electric and Magnetic Field Mapping With the pnCCD (S)TEM Camera
}

\author{
M. Huth ${ }^{1}$, S. Ihle ${ }^{1}$, R. Ritz ${ }^{1}$, M. Simson ${ }^{1}$, H. Soltau ${ }^{1}$, V. Migunov ${ }^{2}$, M. Duchamp ${ }^{2}$, R.E. Dunin-
} Borkowski ${ }^{2}$, H. Ryll ${ }^{3}$ and L. Strüder ${ }^{3}$

1. PNDetector GmbH, Otto-Hahn-Ring 6, München, Germany

2. Ernst Ruska-Centre for Microscopy and Spectroscopy with Electrons, FZJ, Jülich, Germany

3. PNSensor GmbH, Otto-Hahn-Ring 6, München, Germany

For the research on modern materials and devices and their properties, it is of great interest to gain information about local electric and magnetic fields on a nanometer scale. Therefore, techniques providing a fast, direct and precise measurement of local fields are useful for materials science applications. We present microscopic measurements of electric and magnetic fields with the 4D-STEM technique using the pnCCD (S)TEM camera. In 4D-STEM, a 2D camera image is recorded for each probe position of a $2 \mathrm{D}$ scan area, yielding a $4 \mathrm{D}$ dataset. With this technique, small shifts of the bright field disc (BFD) due to a deflection of the electron beam through electric and magnetic fields in the sample region can be detected. Hence, magnitude and direction of the local field at each probe position can be determined. Given the large number of necessary probe positions, this technique requires a fast camera system providing short enough readout times so that instabilities in the microscope and sample drift do not deteriorate the final STEM image as well as reasonable acquisition times. Furthermore, a pixelated detector is required to record and account for the intensity distribution variations caused by interaction of the electron beam with the sample.

The pnCCD (S)TEM camera provides a fast acquisition of 2D camera images with a direct detecting, radiation hard pnCCD with $264 \times 264$ pixels [1]. Routinely, the readout speed is 1000 frames per second (fps) and can be further increased through binning and windowing. For example, with the pnCCD (S)TEM camera a $256 \times 256$ STEM image with 65,536 probe positions - where a camera image is recorded at each probe position - can be recorded in less than $70 \mathrm{~s}$. The $264 \times 264$ pixel camera image allows a precise determination of the BFD position yielding information about the electric and magnetic fields in the sample. Image subsets can be selected freely for data analysis allowing a flexible postexperiment selected area diffraction or differential phase contrast (DPC) analysis. A major advantage over conventional segmented DPC detectors is that, with the pnCCD (S)TEM camera, movements of the BFD can be discriminated from intensity variations inside the BFD. Further 4D-STEM applications benefitting from the pnCCD (S)TEM camera include imaging on the micro- and millisecond timescale [2], strain analysis [3], magnetic domain mapping, and electron ptychography [4].

A demonstration of electrical field mapping with the pnCCD (S)TEM camera is shown in Figure 1. A voltage of $50 \mathrm{~V}$ was applied to a tungsten needle mounted in an FEI Titan 80-200 microscope, operated at $80 \mathrm{keV}$. For each of the $256 \times 256$ probe positions, a 2D camera image was recorded (Fig. 1a). From these camera images an incoherent bright field STEM image (Fig. 1b, background) as well as the position in $\mathrm{x}$ - and $\mathrm{y}$-direction of the BFD at each probe position was calculated. A comparison of the BFD position of a measurement with and without applied voltage yields information about magnitude and direction of the local gradient of the projected electrostatic potential (Fig. 1b, indicated by coloring and arrows). In addition to this direct mapping of the electrical field around a needle with rather wellshaped BFDs, the large number of pixels of the pnCCD (S)TEM camera allows the precise determination of the BFD position, even in cases when the BFD is weak and deformed through the 
interaction of the electron beam with the sample (Fig. 1c). A comparable mapping of magnetic fields is also possible and was demonstrated with a Lorentz-like STEM setup on a nickel sample.

In conclusion, 4D-STEM techniques like electric and magnetic field mapping benefit significantly from the capabilities of the pnCCD (S)TEM camera. The readout speeds of $1000 \mathrm{fps}$ and above allow the fast acquisition of 4D datasets with 2D camera images at each probe position. Through the large number of pixels, position and intensity variations of BFDs can be precisely determined.

\section{References:}

[1] H Ryll et al, Microscopy and Microanalysis 19 (2013), p.1160-1161.

[2] H Ryll et al, Microscopy and Microanalysis 21 (Suppl. 3) (2015), p. 1585-1586.

[3] K Müller et al, Appl. Phys. Lett. 101 (2012), p. 2121101-2121104.

[4] H Yang et al, Microscopy and Microanalysis 21 (Suppl. 3) (2015), p. 2303-2304.

(a)

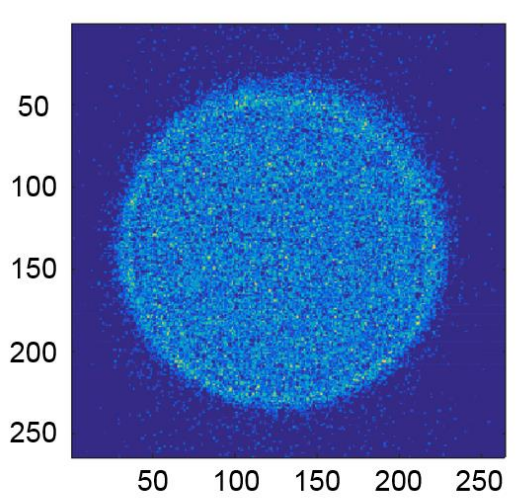

(c)

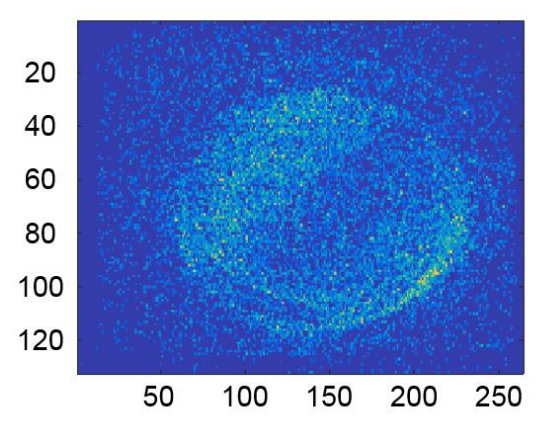

(b)

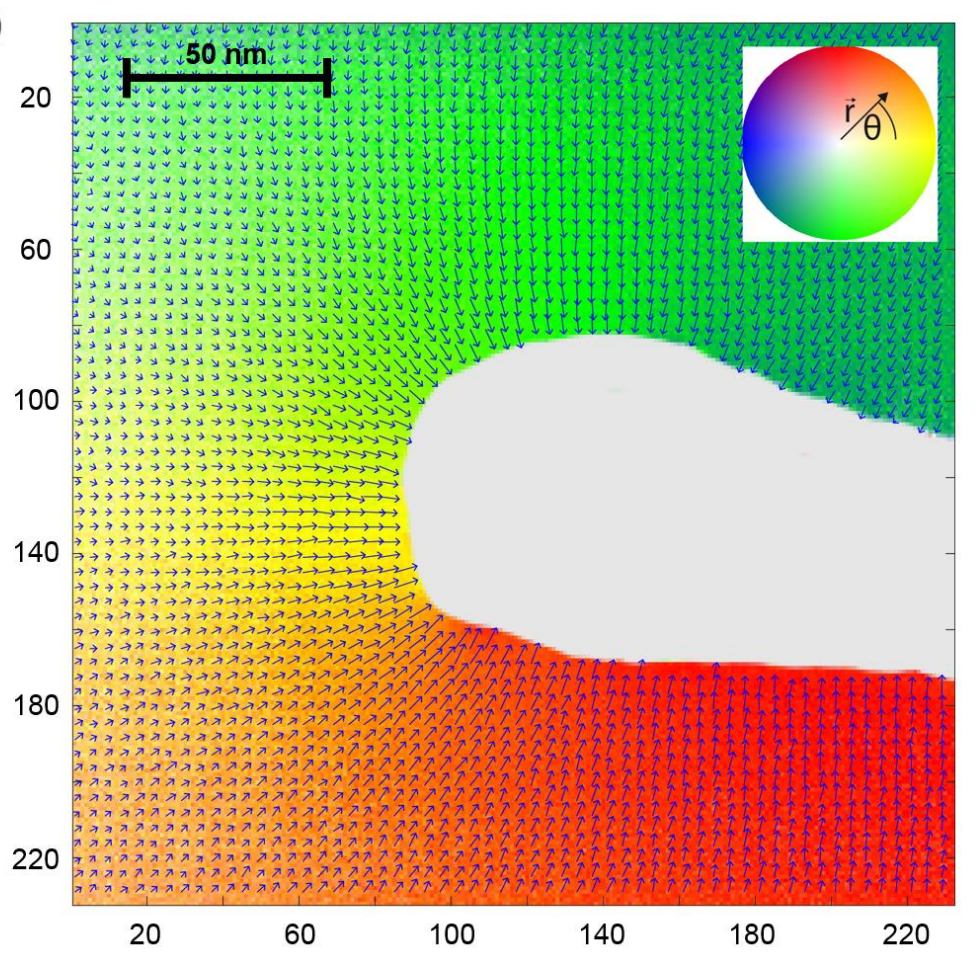

Figure 1: (a) Single camera image at one probe position recorded with a full frame readout at a speed of 1000 frames per second (fps). The camera image with 264x264 pixels allows the precise determination of the position of the bright field disc (BFD). (b) STEM image of a tungsten needle. A voltage of $50 \mathrm{~V}$ was applied between the needle and a counter-electrode in a distance of $\approx 100 \mu \mathrm{m}$. Superimposed is a vector field indicating direction and magnitude of the local electric field at the probe positions outside the needle. The area of the needle is shown grayed. The direction of the vectors is additionally indicated by the coloring in the background. The vector field was calculated from movements of the position of the BFD between a measurement with $(50 \mathrm{~V})$ and without $(0 \mathrm{~V})$ applied voltage. The length of the arrows indicates the shift of the BFD, which is proportional to the local gradient of the projected electrostatic potential. (c) Single camera image of a BFD recorded with a solar-cell as sample. A twofold binning at a readout speed of $2000 \mathrm{fps}$ was used. The BFD is blurred through interactions of the sample with the electron beam. Due to the large number of pixels of the pnCCD (S)TEM camera, the BFD position can be determined precisely nevertheless. 